\title{
The loud and the quiet: searching for radio counterparts of two radio-weak BL Lac candidates with VLBI
}

\author{
Hong-Min Cao, ${ }^{1,2 \star}$ Sándor Frey, ${ }^{3}$ Krisztina É. Gabányi, ${ }^{3,4}$ Jun Yang, ${ }^{5}$ Lang Cui, ${ }^{1}$ \\ Xiao-Yu Hong ${ }^{6}$ and Tao $\mathrm{An}^{6}$ \\ ${ }^{1}$ Xinjiang Astronomical Observatory, Chinese Academy of Sciences, 150 Science 1-Street, Urumqi, Xinjiang 830011, China \\ ${ }^{2}$ School of Electronic and Electrical Engineering, Shangqiu Normal University, Wenhua Road 298, Shangqiu, Henan 476000, China \\ ${ }^{3}$ Konkoly Observatory, MTA Research Centre for Astronomy and Earth Sciences, Konkoly Thege Miklós út 15-17, H-1121 Budapest, Hungary \\ ${ }^{4}$ MTA-ELTE Extragalactic Astrophysics Research Group, Pázmány Péter sétány 1/A, H-1117 Budapest, Hungary \\ ${ }^{5}$ Department of Space, Earth and Environment, Chalmers University of Technology, Onsala Space Observatory, SE-43992 Onsala, Sweden \\ ${ }^{6}$ Shanghai Astronomical Observatory, Key Laboratory of Radio Astronomy, Chinese Academy of Sciences, 80 Nandan Road, Shanghai 200030, \\ China
}

Accepted 2018 XXX NN. Received 2018 XXX NN; in original form 2018 July 26

\begin{abstract}
BL Lac objects are known to have compact jets inclined to our line of sight at a small angle, showing prominent radio emission. Two radio-weak BL Lac candidates with no counterparts in current radio surveys were recently reported by Massaro et al. (2017). Both sources were selected as candidate low-energy counterparts of unassociated Fermi $\gamma$-ray sources. We carried out very long baseline interferometry (VLBI) observations with the European VLBI Network (EVN) at $5 \mathrm{GHz}$ to explore their radio properties at milli-arcsecond (mas) scale. One target, J1410+7405, is clearly detected with the EVN. Its measured 5-GHz flux density, $2.4 \mathrm{mJy}$, is consistent with recent interferometric measurements with the Karl G. Jansky Very Large Array, suggesting that the radio emission is confined to the inner $\lesssim 10$ mas region. J1410+7405 is therefore identified as a radio-loud jetted active galactic nucleus, and its brightness temperature exceeds $\sim 10^{9} \mathrm{~K}$. Its properties are similar to those of other $\gamma$-ray detected BL Lac objects. On the other hand, the second target, J0644+6031, remains undetected with the EVN with a $6-\sigma$ brightness upper limit of $0.12 \mathrm{mJy}$ beam $^{-1}$. This source is thus radio-quiet, confirming its peculiarity, or possibly questioning its BL Lac nature.
\end{abstract}

Key words: galaxies: active - BL Lacertae objects: general - quasars: general galaxies: individual: J0644+6031 - galaxies: individual: J1410+7405

\section{INTRODUCTION}

Blazars, a sub-class of active galactic nuclei (AGN), produce prominent non-thermal emission across the entire electromagnetic spectrum. This emission could even dominate over the thermal components coming from the accretion disk, the obscuring torus and the host galaxy. Blazars typically exhibit flux density variability on multiple time scales, from minutes to years, and often show polarization in radio and optical. All these observing characteristics are ascribed to the twin relativistic jets closely aligned with the line of sight, launched from the AGN central engine, an accreting supermassive black hole. The approaching jet con-

* E-mail: hongmin.cao@foxmail.com (H-MC) tributes the most due to the Doppler-boosting effect (see Urry \& Padovani 1995, for a review).

Blazars are traditionally divided into two main subclasses: BL Lac objects and flat-spectrum radio quasars (FSRQs). The former have no or weak optical emission lines, while the latter have pronounced emission lines. The rest-frame equivalent width (REW) of $5 \AA$ is usually considered as the dividing line between these two source populations (e.g. Stickel et al. 1991). The broadband $\log v F_{v}-\log v$ spectral energy distributions (SEDs) of blazars show a double-hump feature which can be generally explained in the context of the one-zone leptonic model (Ghisellini \& Tavecchio 2009). Blazars are the dominant emitters in the $\gamma$-ray sky. Among the identified/associated Fermi sources in the third Large Area Telescope (LAT) 
source catalogue (3FGL), about 57 per cent are blazars (Acero et al. 2015).

In the Wide-field Infrared Survey Explorer (WISE) midinfrared (mid-IR) color-color diagram, blazars appear to be located in a region different from those occupied by thermal sources. This is the WISE blazar strip (WBS), where the thermal emission is contaminated by the non-thermal emission produced by the relativistic jet (Massaro et al. 2011). The region of the $\gamma$-ray blazars is even narrower, and is called the WISE gamma-ray strip (WGS, D'Abrusco et al. 2012; Massaro et al. 2012). The WISE mid-IR colors falling into the WGS are widely considered as a piece of evidence for classifying a source as a blazar.

Very recently, Massaro et al. (2017) identified two sources, WISE J064459.38+603131.7 (hereafter J0644+6031) and WISE J141046.00+740511.2 (hereafter $\mathrm{J} 1410+7405)$, as genuine radio-weak BL Lacs (RWBLs) ${ }^{1}$. They are particularly interesting because they have no radio counterparts at $1.4 \mathrm{GHz}$ in the U.S. National Radio Astronomy Observatory (NRAO) Very Large Array (VLA) Sky Survey ${ }^{2}$ (NVSS, Condon et al. 1998). Both sources are outside the sky coverage of the VLA Faint Images of the Radio Sky at Twenty-centimeters ${ }^{3}$ survey (FIRST, White et al. 1997). Radio emission is usually seen as a representative characteristic of blazars with relativistic jets. It is thus puzzling why these two alleged BL Lacs remained undetected in radio. Are they really silent, or do they have radio emission which is unknown just because it is below the detection limit $(\sim 2.5 \mathrm{mJy})$ of the NVSS?

We observed J0644+6031 and J1410+7405 using the technique of very long baseline interferometry (VLBI) with the European VLBI Network (EVN) at $5 \mathrm{GHz}$. These VLBI observations were sufficiently sensitive to detect compact emission if the sources are radio-loud. VLBI can provide direct proof of the blazar nature of an AGN by means of detecting milli-arcsecond (mas) scale radio emission originating from the inner jet. The aim of our EVN experiment was thus confirming or ruling out that these two intriguing objects are BL Lacs.

The observed characteristics of the two targets are introduced in Section 2. The details of the VLBI experiment and the data reduction are described in Section 3. The results are presented in Section 4 and discussed in Section 5. A summary is given in Section 6. In this letter, we use a flat $\Lambda \mathrm{CDM}$ model with $H_{0}=71 \mathrm{~km} \mathrm{~s}^{-1} \mathrm{Mpc}^{-1}, \Omega_{\mathrm{m}}=0.27$ and $\Omega_{\Lambda}=0.73$ (Spergel et al. 2007) for cosmological distance estimation.

\section{TARGET SOURCES}

J0644+6031 was selected as a blazar candidate counterpart of 2FGL J0644.6+6034 (Massaro et al. 2013), an unassociated $\gamma$-ray source (UGS) in the second Fermi LAT source

\footnotetext{
1 Here the term "radio-weak" means no radio counterparts known in the current major radio surveys, thus low radio flux density. However, Bruni et al. (2018) adopt 1.4-GHz monochromatic radio power $L_{1.4}<10^{25.5} \mathrm{~W} \mathrm{~Hz}^{-1}$, suggested by Gregg et al. (1996) for radio-quiet AGN, to define RWBLs.

2 https://www.cv.nrao.edu/nvss/

3 http://sundog.stsci.edu/
}

catalogue (2FGL, Nolan et al. 2012). The nearest USNOB (Monet et al. 2003) optical counterpart to this WISE source is just $0 . \prime 3$ away $^{4}$. Follow-up optical spectroscopic observations by Paggi et al. (2014) revealed weak emission lines with REWs consistent with the threshold of $5 \AA$, supporting the BL Lac classification, and suggest a redshift of $z=0.3582 \pm 0.0008$.

$\mathrm{J} 1410+7405$ is coincident with one of the two X-ray sources found in the position error ellipse of an UGS 1FHL J1410.4+7408 (Landi et al. 2015), listed in the first Fermi LAT high-energy source catalogue (1FHL, Ackermann et al. 2013). Only one USNO-B optical source, 0."4 away from $\mathrm{J} 1410+7405$, is located in the error circle of the X-ray source $\left(\sim 6^{\prime \prime}\right.$ in radius, Landi et al. 2015). It has an optical spectrum typical of BL Lacs, barely showing any emission or absorption lines and therefore prohibiting redshift determination (Marchesini et al. 2016).

Both WISE sources (J0644+6031 and J1410+7405) lie in the subregion on the WGS populated by the $\gamma$-ray BL Lacs. They also show flux density variation known from their light curves built from the WISE single-epoch photometric observations covering $\sim 2$ days each. Moreover, the multi-band photometric data of these two sources can be well described by a log-parabolic function often used to fit the non-thermal SEDs of BL Lacs. The properties of their optical spectra, mid-IR colors and variability, and the shape of their broad-band SEDs together suggest the blazar nature of these two WISE sources (Massaro et al. 2017).

\section{OBSERVATIONS AND DATA REDUCTION}

\subsection{Observations}

The 5-GHz EVN experiment, divided into two segments with project codes EC061A and EC061B, was conducted on 2017 October 26. There were thirteen participating antennas in both segments: Effelsberg (Ef, Germany), Jodrell Bank Mk2 (Jb, United Kingdom), Medicina (Mc, Italy), Toruń (Tr, Poland), Westerbork (Wb, the Netherlands), Noto (Nt, Italy), Yebes (Ys, Spain), Tianma (T6, China), Urumqi (Ur, China), Svetloe (Sv, Russia), Badary (Bd, Russia), Zelenchukskaya (Zc, Russia), and Irbene (Ir, Latvia). For most of the antennas, the data were recorded at $2048 \mathrm{Mbps}$ rate, with 2 polarizations, 8 intermediate frequency channels (IFs) per polarization, and $32 \mathrm{MHz}$ bandwidth per IF. For Jb, Tr, and $\mathrm{Wb}, 4$ IFs per polarization were used, with a recording rate of $1024 \mathrm{Mbps}$. The duration of each segment was about $2 \mathrm{~h}$. The observations were carried out in phase-referencing mode (e.g. Beasley \& Conway 1995), i.e., the antennas nodded between the nearby bright and compact phase calibrator and the target. The cycle time was 5-min long, with an ontarget time of $3.5 \mathrm{~min}$ in each cycle. Short 5-min scans of bright fringe-finder sources (J1419+5423 and DA193 for segments EC061A and EC061B, respectively) were also scheduled. The recorded data from the individual stations were correlated at the Joint Institute for VLBI ERIC (JIVE) in Dwingeloo, the Netherlands, with $2 \mathrm{~s}$ integration time and 64 spectral channels per IF. Further observing information

${ }^{4}$ http://vizier.u-strasbg.fr/viz-bin/VizieR-3?-source=I/284 
Table 1. EVN observing information.

\begin{tabular}{lccccccc}
\hline \hline Project segment & Target & $\begin{array}{c}\text { Right ascension } \\
\mathrm{h} \mathrm{m} \mathrm{s}\end{array}$ & $\begin{array}{c}\text { RA err. } \\
\text { mas }\end{array}$ & $\begin{array}{c}\text { Declination } \\
\text { / / }\end{array}$ & $\begin{array}{c}\text { Dec err. } \\
\text { mas }\end{array}$ & $\begin{array}{c}\text { Phase calibrator } \\
\text { Separation } \\
\circ\end{array}$ \\
\hline EC061A & $\mathrm{J} 1410+7405$ & 141045.9505 & 4.5 & +740510.905 & 4.1 & $\mathrm{~J} 1353+7532$ & 1.85 \\
EC061B & $\mathrm{J} 0644+6031$ & 064459.3851 & 1.4 & +603131.655 & 1.6 & $\mathrm{~J} 0650+6001$ & 0.85 \\
\hline
\end{tabular}

Notes: Cols. 3-6 - a priori equatorial coordinates (J2000) assumed for pointing to the target sources and their errors, taken from the Gaia DR1 catalogue (Gaia Collaboration 2016a,b); Col. 8 - angular separation between the target and the phase calibrator.

is given in Table 1. In this experiment, the Gaia Data Release 1 (DR1) coordinates ${ }^{5}$ (Gaia Collaboration 2016a,b) of the optical counterparts of the target sources were used for pointing the radio telescopes and for the correlation.

\subsection{Data reduction}

The VLBI data were calibrated in the NRAO Astronomical Image Processing System (AIPS, Greisen 2003) in both segments separately, according to the EVN Data Reduction Guide $^{6}$. A priori amplitude calibration was carried out using the known antenna gain curves and system temperatures regularly measured at the VLBI stations during the observations, or the nominal system equivalent flux densities where the system temperatures were not available. Parallactic angle and ionospheric corrections were then performed successively. Manual phase calibration, global fringe-fitting and bandpass calibration were also done.

The calibrated visibility data of the phase-reference and fringe-finder sources were exported to the DIFMAP program (Shepherd 1997) for hybrid mapping. The antenna-based amplitude correction factors obtained by the GSCALE command were fed back into AIPS using the task CLCOR, to adjust the visibility amplitudes for the target sources as well. The factors lower than \pm 5 per cent were not applied but corrections were typically higher. Fringe-fitting was then repeated in AIPS for the phase-reference calibrators, but this time their brightness distribution models produced in DIFMAP were taken into account. This eliminates the influence of the structure that may cause residual phase errors. Finally, the solutions obtained for the reference sources were interpolated to the targets in AIPS, and the phase-referenced calibrated visibility data were analysed in DIFMAP.

\section{RESULTS}

$\mathbf{J 1 4 1 0}+\mathbf{7 4 0 5}$ is clearly detected with VLBI. A single circular Gaussian model component was used to fit the source brightness distribution in the visibility domain in DIFMAP. Due to the weakness of the source, self-calibration was not attempted. The naturally-weighted modelfit image is shown in Fig. 1. VLBI phase-referencing allows us to measure the accurate J2000 coordinates of the radio brightness peak in $\mathrm{J} 1410+7405$. The right ascension $\mathrm{RA}=14^{\mathrm{h}} 10^{\mathrm{m}} 45^{\mathrm{s}} .95002$ and declination $\mathrm{DEC}=+74^{\circ} 05^{\prime} 10^{\prime \prime}$ ' 9061 were determined from the

\footnotetext{
5 https://gea.esac.esa.int/archive/

6 http://www.evlbi.org/user_guide/analysis.html
}

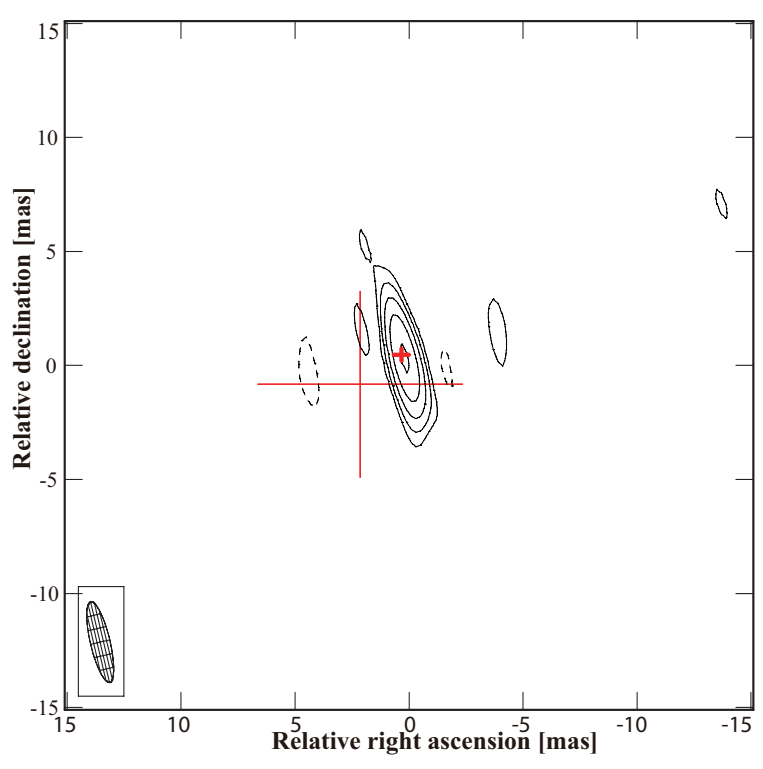

Figure 1. Phase-referenced VLBI image of J1410+7405 at $5 \mathrm{GHz}$, made with natural weighting and centred on the peak position. The first contours are drawn at $\pm 129 \mu \mathrm{Jy}_{\mathrm{beam}}{ }^{-1}( \pm 5 \sigma$ image noise level). The positive contours increase by a factor of 2 . The peak brightness is $2.2 \mathrm{mJy}$ beam $^{-1}$. The Gaussian restoring beam with 3.6 mas $\times 0.9$ mas $(F W H M)$ is shown in the lower left corner. The position angle of the beam major axis is $13^{\circ}$, measured from north through east. The crosses denote the source optical positions taken from Gaia DR1 (Table 1) and Gaia DR2 (see the text), with their sizes indicating the uncertainties.

image using the AIPS task TVMAXFIT. The estimated positional uncertainty of $\sim 0.2$ mas is dominated by the systematic error components, i.e., the positional uncertainty of the phase-reference calibrator $\mathrm{J} 1353+7532$, and the uncertainty caused by the target-calibrator angular separation (Pradel et al. 2006). The statistical error is negligible in this case, due to the high dynamic range of the phasereferenced image. The Gaia DR1 optical position (see Table 1 and Fig. 1) is consistent with that measured with VLBI within the errors, which strengthens the physical association of the optical and radio source. Note that the most recent Gaia DR2 (Gaia Collaboration 2018) optical position $\mathrm{RA}=14^{\mathrm{h}} 10^{\mathrm{m}} 45^{\mathrm{s}} .95006$ and $\mathrm{DEC}=74^{\circ} 05^{\prime} 100^{\prime \prime} 9063$ (uncertainty $\sim 0.3$ mas, Fig. 1) is also consistent with and is even closer to our VLBI position.

The measured parameters of $\mathrm{J} 1410+7405$ are listed in Table 2. The brightness temperature $T_{\mathrm{b}}$ in the source rest frame is calculated as

$T_{\mathrm{b}}=1.22 \times 10^{12} 1+z \frac{S_{V}}{\theta^{2} v_{\mathrm{obs}}^{2}} \mathrm{~K}$ 
Table 2. Parameters of the targets derived from the 5-GHz EVN observations.

\begin{tabular}{cccc}
\hline \hline Source name & $\begin{array}{c}S_{V} \\
\mathrm{mJy}\end{array}$ & $\begin{array}{c}\theta \\
\operatorname{mas}\end{array}$ & $\begin{array}{c}T_{\mathrm{b}} \\
10^{9} \mathrm{~K}\end{array}$ \\
\hline $\mathrm{J} 1410+7405$ & $2.4 \pm 0.4$ & $0.28 \pm 0.01$ & $>1.2$ \\
$\mathrm{~J} 0644+6031$ & $<0.12$ & - & - \\
\hline
\end{tabular}

Notes: Col. 2 - flux density; Col. 3 - circular Gaussian model component diameter (FWHM); Col. 4 - brightness temperature. Modelfit errors are estimated according to Lee et al. (2016)

based on the 3- $\sigma$ image noise level. For the flux density, additional 10 per cent error was considered, to account for the VLBI amplitude calibration uncertainty.

(Condon et al. 1982), where $z$ is the redshift, $S_{V}$ the observed flux density in Jy, $\theta$ the Gaussian component size (full width at half maximum, FWHM) in mas, and $v_{\text {obs }}$ the observing frequency in GHz. Since the redshift of J1410+7405 is unknown, we derived a lower limit $\left(T_{\mathrm{b}}>1.2 \times 10^{9} \mathrm{~K}\right)$ assuming $z=0$. This value suggests that the radio emission originates from a jet and is produced by non-thermal synchrotron radiation. The fitted component size $(0.28 \pm 0.01$ mas, Table 2$)$ is slightly smaller than the minimum resolvable size (e.g. Kovalev et al. 2005), 0.31 mas, indicating the source is unresolved. The latter value was used to calculate the brightness temperature. The strong phase coherence on one of the longest and the most sensitive Ef-T6 baseline confirms the compactness of J1410+7405.

In case of our other target, J0644+6031, we searched for compact radio emission in the naturally weighted dirty map with a field of view as large as $2^{\prime \prime} \times 2^{\prime \prime}$, even though the a priori Gaia position was accurate to mas level (Table 1). However, nothing was detected above a signal-to-noise ratio of 6 . The $6-\sigma$ brightness upper limit is $120 \mu \mathrm{Jy}_{\text {beam }}{ }^{-1}$. We conclude that any mas-scale compact radio source in J0644+6031 should be weaker than 0.12 mJy (Table 2).

We repeated the analysis to constrain the VLBI network to relatively shorter baselines by removing data from T6, $\mathrm{Ur}$ and $\mathrm{Bd}$, to become more sensitive to $\sim 10$ mas scale extended radio emission. For J1410+7405, one circular Gaussian component fitting gave a flux density of $2.5 \pm 0.5 \mathrm{mJy}$, consistently with that measured with the full VLBI array (Table 2), which is another support for the source compactness. However, no radio source was found for J0644+6031.

\section{DISCUSSION}

Among the Fermi $\gamma$-ray sources, a large fraction still have no known counterparts in other electromagnetic wavebands. For example, in 3FGL, about 30 per cent remain to be identified/associated (Acero et al. 2015). Finding their low-energy counterparts is essential to reveal the physical origin of the $\gamma$-ray emission, and thus to better understand the whole $\gamma$ ray sky. Considering that a large percentage of the associated Fermi sources are blazars, if there is a blazar discovered within the positional error ellipse (normally at 95 per cent confidence level) of an UGS, then this blazar would be the most likely object where the $\gamma$-rays come from.

The compact radio emission detected with VLBI proves the existence of jet activity in J1410+7405. This
mid-IR source is positionally coincident with UGS 1FHL J1410.4+7408 (a.k.a. UGS 3FGL J1410.9+7406, Landi et al. 2015; Massaro et al. 2017). Based on the multi-band data available so far, J1410+7405 is the most promising $\gamma$-ray emitter in the Fermi error ellipse.

This source is also detected with the Karl G. Jansky VLA at $5 \mathrm{GHz}(2.2 \pm 0.1 \mathrm{mJy})$ and $7 \mathrm{GHz}(2.0 \pm 0.1 \mathrm{mJy})$, in a large observing project carried out very recently, aiming to search for radio counterparts of all UGSs in 3FGL (Schinzel et al. 2017). The deduced flat spectrum with spectral index $\alpha=-0.28 \pm 0.28$ is consistent with a compact synchrotron self-absorbed radio source (the spectral index is defined as $S_{v} \propto v^{\alpha}$ ). Our $5-\mathrm{GHz}$ VLBI flux density $(2.4 \pm 0.4 \mathrm{mJy})$ is identical within the errors with that measured by the VLA, suggesting that the radio emission essentially comes from a small mas-scale region probed by the EVN. In addition, the accurate match of the source positions measured with VLBI and Gaia, and the high compactness (the ratio of the VLBI and VLA flux densities close to 1) imply that the jet is oriented at a small viewing angle (e.g. Liuzzo et al. 2013). However, the VLA experiment (Schinzel et al. 2017) was carried out more than 2 years before our EVN observations. We therefore cannot rule out flux density variability in $\mathrm{J} 1410+7405$ which is typical for compact blazars.

The relative strength of AGN jet activity could be characterised with the radio-loudness parameter $R=S_{\mathrm{r}} S_{\mathrm{o}}$. Here, $S_{\mathrm{r}}$ and $S_{\mathrm{o}}$ denote the observed flux densities at $5 \mathrm{GHz}$ and $4400 \AA$, respectively (Kellermann et al. 1989). Alternatively, the rest-frame flux density $\left(S_{v, \text { rest }} \propto \frac{v^{\alpha}}{1+z^{1+\alpha}}\right)$ is also used to calculate the radio loudness (e.g. Wu et al. 2013). As the redshift (for J1410+7405) and the optical spectral indices (for both targets) are not known, we used the classical definition of radio loudness (Kellermann et al. 1989) with observed flux densities, but this will not change our conclusions. AGN are traditionally classified as radio-quiet if $R<10$ and radio-loud if $R \geq 10$. Known from the USNO-A2.0 catalogue $^{7}$ (Monet et al. 1998), the optical B-band magnitude of $\mathrm{J} 1410+7405$ is 19.2 . This, together with the $5-\mathrm{GHz}$ radio flux density, gives $R \approx 40$, indicating that $\mathrm{J} 1410+7405$ is radio-loud.

Taking the median redshift value of 1FHL BL Lac objects, i.e., $z=0.2$ (Lico et al. 2016), we estimate the $1.4-\mathrm{GHz}$ monochromatic radio power $L_{1.4}=3.1 \pm 0.3 \times 10^{23} \mathrm{~W} \mathrm{~Hz}^{-1}$ for $\mathrm{J} 1410+7405$. This value is compatible with the RWBL definition adopted by Bruni et al. (2018). However, if the source is at $z \geq 1.7$, its power $L_{1.4} \geq 10^{25.5} \mathrm{~W} \mathrm{~Hz}^{-1}$ would classify it as radio-loud according to Gregg et al. (1996), consistently with our radio-loudness estimation by the Kellermann et al. (1989) criterion.

The jet Doppler factor $\delta$ can be inferred from a single-epoch VLBI observation, i.e. $\delta=T_{\mathrm{b}} T_{\mathrm{eq}}$, where $T_{\mathrm{eq}} \approx$ $5 \times 10^{10} \mathrm{~K}$ is the equipartition brightness temperature (Readhead 1994). A Doppler factor larger than unity would indicate Doppler-boosted emission and is often used as an important criterion for classifying a source as a blazar (e.g. Coppejans et al. 2016). The measured brightness temperature of $\mathrm{J} 1410+7405\left(T_{\mathrm{b}}>1.2 \times 10^{9} \mathrm{~K}\right)$ is only a lower limit and thus the Doppler factor is $\delta \gtrsim 0.02$. Even if a non-zero

\footnotetext{
${ }^{7}$ http://vizier.u-strasbg.fr/viz-bin/VizieR-3?-source=I/252
} 
redshift is assumed in Eq. 1, the low limit of the core brightness temperature does not usefully constrain the Doppler factor in our case, leaving the question open whether the jet emission in J1410+7405 is not boosted or simply the angular resolution is insufficient to measure $\delta \gtrsim 1$. The core can be blended with a more extended jet component, resulting in an overestimated angular size and thus an underestimation of the source brightness temperature (Natarajan et al. 2017).

The $\gamma$-ray blazars in the Roma-BZCAT $T^{8}$ (Massaro et al. 2015) show a bimodal feature in the distribution of the logarithmic flux density ratio between $1.4 \mathrm{GHz}$ in the radio and $3.4 \mu \mathrm{m}$ in mid-IR (see Fig. 10 in Massaro et al. 2017). The available radio data of $\mathrm{J} 1410+7405$ allow us to calculate $\log S_{1.4 \mathrm{GHz}} S_{3.4 \mu \mathrm{m}}=1.0$, which is different from the value below 0.5 as expected by Massaro et al. (2017), and places the source to the region where $\gamma$-ray BL Lacs populate the distribution.

Our second VLBI target, J0644+6031, is positionally consistent with UGS 2FGL J0644.6+6034, but lies outside the 95 per cent confidence positional error ellipse of UGS 3FGL J0644.6+6035, which has a somewhat more accurate position (see Fig. 8 in Massaro et al. 2017). Thus its physical association with the $\gamma$-ray source is rather uncertain. However, not all known blazars appear detected by Fermi LAT (Lister et al. 2015). Our 5-GHz flux density upper limit (120 $\mu \mathrm{Jy}$; Table 2 ) for the mas-scale compact radio emission and the optical B-band magnitude $(\operatorname{USNO}(B)=19$, Monet et al. 1998) provide a stringent upper limit of $R \lesssim 1.5$ for the radio loudness of J0644+6031. Assuming spectral index $\alpha=0$, we obtain $L_{1.4}<3.8 \times 10^{22} \mathrm{~W} \mathrm{~Hz}^{-1}$ and $\log S_{1.4 \mathrm{GHz}} S_{3.4 \mu \mathrm{m}}<-0.86$ for J0644+6031. The radio non-detection itself and the radio quietness confirm the unusual characteristics of J0644+6031 as a BL Lac object suggested by Massaro et al. (2017). From our EVN data, there is no evidence for a radio jet in this source. However, it is also possible that the BL Lac identification of J0644+6031 is questionable. In this case, the BL Lac-type characteristics in wavebands other than the radio would call for alternative explanations. Indeed, J0644+6031 has a noisier optical spectrum than J1410+7405 (Paggi et al. 2014; Marchesini et al. 2016). A spectrum like this could be caused by the intrinsically weak broad emission lines, rather than those swamped by the continuum emission from the relativistic jet (Plotkin et al. 2010). Based on the data currently available, the nature of J0644+6031 remains elusive, and further efforts are required to draw a final conclusion.

The broadband SEDs of the radio source J1410+7405 and the radio-undetected J0644+6031 are shown in Fig. 2. We calculated the WISE, 2MASS, and Swift UVOT/XRT fluxes following Massaro et al. (2017). The USNO-A2.0 B magnitude was first transformed to the Landolt magnitude 9 and then corrected with the Galactic extinction according to Schlafly \& Finkbeiner (2011). The $\gamma$-ray fluxes and the Fermi 4-year sensitivity curves were obtained with the web-based tool provided by the ASI Science Data Center $(\mathrm{ASDC})^{10}$. The synchrotron peak frequencies derived from

\footnotetext{
8 http://www.asdc.asi.it/bzcat/

9 http://www.quasars.org/docs/USNO_Landolt.htm

10 https://tools.ssdc.asi.it/
}

the log-parabolic fit (see Fig. 2) are $8.2 \pm 2.2 \times 10^{14} \mathrm{~Hz}$ and $1.0 \pm 0.3 \times 10^{14} \mathrm{~Hz}$ for $\mathrm{J} 1410+7405$ and J0644+6031, respectively. Thus, regarding the blazar classification by Abdo et al. (2010), the former source should belong to the intermediate-synchrotron peaked (ISP) BL Lacs, while J0644+6031, if indeed a BL Lac, would be around the dividing line between the ISP and low-synchrotron peaked (LSP) objects.

\section{SUMMARY AND CONCLUSIONS}

We observed with the EVN two radio-weak BL Lac candidates, proposed as counterparts of unidentified Fermi $\gamma$ ray sources (Massaro et al. 2017). Our 5-GHz observations capable of mas-scale angular resolution did not detect any compact radio source at the position of J0644+6031. The inferred small value of radio-loudness parameter $R \lesssim 1.5$ confirms the peculiarity of this object found by Massaro et al. (2017). It might either be a true RWBL or its BL Lac identification should be revised since this class of objects is known to show synchrotron radio emission from relativistic jets closely aligned to the line of sight. Both the BL Lac nature of J0644+6031 and its association with a Fermi source would require further supporting evidence.

On the other hand, compact radio emission from the source J1410+7405 is detected with VLBI. The measured brightness temperature of the 2.4-mJy component is in the order of $10^{9} \mathrm{~K}$, not sufficient to prove that the jet emission is Doppler-boosted. Nevertheless, its properties are similar to those of other known 1 FHL BL Lac objects. The radio loudness of the source is $R \approx 40$. The case of J1410+7405 demonstrates that the lack of detection in a relatively shallow radio survey like the NVSS does not necessarily preclude the existence of mas-scale compact radio emission in a radioloud (jetted) AGN.

\section{ACKNOWLEDGEMENTS}

We thank the anonymous referee for valuable suggestions. The European VLBI Network is a joint facility of independent European, African, Asian, and North American radio astronomy institutes. Scientific results from data presented in this publication are derived from the following EVN project code: EC061. H-MC acknowledges support by the CAS "Light of West China" programme (Grants No. 2016-QNXZ-B-21 \& YBXM-2014-02) and the National Natural Science Foundation of China (Grants No. U1731103, 11573016 and 11503072). SF and KÉG thank the Hungarian National Research, Development and Innovation Office (OTKA NN110333) for support. KÉG was supported by the János Bolyai Research Scholarship of the Hungarian Academy of Sciences. This work has made use of data from the European Space Agency (ESA) mission Gaia (https://www.cosmos.esa.int/gaia), processed by the Gaia Data Processing and Analysis Consortium (DPAC, https://www . cosmos.esa.int/web/gaia/dpac/consortium). Funding for the DPAC has been provided by national institutions, in particular the institutions participating in the Gaia Multilateral Agreement. 



Figure 2. The broadband SEDs of the two EVN targets J0644+6031 (left panel) and J1410+7405 (right panel). As J0644+6031 is not detected with the EVN, the 5-GHz flux upper limit (marked with $\times$ ) is shown in the left panel. Three $\gamma$-ray data points are plotted to illustrate the Fermi detection. Since the association of J0644+6031 with the Fermi $\gamma$-ray source is uncertain, being outside the 95 per cent confidence positional error ellipse of 3FGL J0644.6+6035 (Massaro et al. 2017), the $\gamma$-ray data points are marked with open circles. The Fermi 4-yr sensitivity curves, based on 3FGL, correspond to a Test Statistic (TS) of 2.

\section{REFERENCES}

Abdo A. A., et al., 2010, ApJ, 708, 1310

Acero F. et al., 2015, ApJS, 218, 23

Ackermann M. et al., 2013, ApJS, 209, 34

Beasley A. J., Conway J. E., 1995, in Zensus J. A., Diamond P. J., Napier P. J., eds, ASP Conf. Ser. Vol. 82., Very Long Baseline Interferometry and the VLBA. Astron. Soc. Pac., San Francisco, p. 327

Bruni G. et al., 2018, ApJ, 854, L23

Condon J. J., Condon M. A., Gisler G., Puschell J. J., 1982, ApJ, 252,102

Condon J. J., Cotton W. D., Greisen E. W., Yin Q. F., Perley R. A., Taylor G. B., Broderick J. J., 1998, AJ, 115, 1693

Coppejans R. et al, 2016, MNRAS, 463, 3260

D’Abrusco R., Massaro F., Ajello M., Grindlay J. E., Smith H. A., Tosti G., 2012, ApJ, 748, 68

Gaia Collaboration, 2016a, A\&A, 595, A1

Gaia Collaboration, 2016b, A\&A, 595, A2

Gaia Collaboration, 2018, A\&A, in press (arXiv:1804.09365)

Ghisellini G., Tavecchio F., 2009, MNRAS, 397, 985

Gregg M. D., Becker R. H., White R. L., Helfand D. J., McMahon R. G., Hook I. M., 1996, AJ, 112, 407

Greisen E. W., 2003, in Heck A., ed, Astrophysics and Space Science Library Vol. 285, Information Handling in Astronomy - Historical Vistas. Kluwer, Dordrecht, p. 109

Kellermann K. I., Sramek R., Schmidt M., Shaffer D. B., Green R., 1989, AJ, 98, 1195

Kovalev Y. Y. et al., 2005, AJ, 130, 2473

Landi R., Bassani L., Stephen J. B., Masetti N., Malizia A., and Ubertini P., 2015, A\&A, 581, A57

Lee S.-S. et al., 2016, ApJS, 227, 8

Lico R., Giroletti M., Orienti M., D’Ammando F., 2016, A\&A, 594, A60

Liuzzo E., et al., 2013, A\&A, 560, A23

Lister M. L., Aller M. F., Aller H. D., Hovatta T., Max-Moerbeck W., Readhead A. C. S., Richards J. L., Ros E., 2015, ApJ,
810, L9

Marchesini E. J. et al., 2016, A\&A, 596, A10

Massaro F., D'Abrusco R., Ajello M., Grindlay J. E., Smith H. A., 2011, ApJ, 740, L48

Massaro F., D'Abrusco R., Tosti G., Ajello M., Gasparrini D., Grindlay J. E., Smith H. A., 2012, ApJ, 750, 138

Massaro F., D'Abrusco R., Paggi A., Masetti N., Giroletti M., Tosti G., Smith H. A., Funk S., 2013, ApJS, 206, 13

Massaro E., Maselli A., Leto C., Marchegiani P., Perri M., Giommi P., Piranomonte S., 2015, Ap\&SS, 357, 75

Massaro F., Marchesini E. J., D'Abrusco R., Masetti N., Andruchow I., Smith H. A., 2017, ApJ, 834, 113

Monet D. G., et al. 1998, USNO-A2.0 catalog, US Naval Obs., Flagstaff

Monet D. G., et al., 2003, AJ, 125, 984

Natarajan I., Paragi Z., Zwart J., Perkins S., Smirnov O., van der Heyden K., 2017, MNRAS, 464, 4306

Nolan P. L. et al., 2012, ApJS, 199, 31

Paggi A. et al., 2014, AJ, 147, 112

Pradel N., Charlot P., Lestrade J. F., 2006, A\&A, 452, 1099

Plotkin R. M. et al., 2010, AJ, 139, 390

Readhead A. C. S., 1994, ApJ, 426, 51

Schinzel F. K., Petrov L., Taylor G. B., Edwards P. G., 2017, ApJ, 838, 139

Schlafly E. F., Finkbeiner D. P., 2011, ApJ, 737, 103

Shepherd M. C., 1997, in Hunt G., Payne H. E., eds, ASP Conf. Ser. Vol. 125, Astronomical Data Analysis Software and Systems VI. Astron. Soc. Pac., San Francisco, p. 77

Spergel D. N. et al., 2007, ApJS, 170, 377

Stickel M., Padovani P., Urry C. M., Fried J. W., Kühr H., 1991, ApJ, 374, 431

Urry C. M., Padovani P., 1995, PASP, 107, 803

Wu J.-F., Brandt W. N., Miller B. P., Garmire G. P., Schneider D. P., Vignali C., 2013, ApJ, 763, 109

White R. L., Becker R. H., Helfand D. J., Gregg M. D., 1997, ApJ, 475, 479 